\title{
No Directed Fractal Percolation in Zero Area
}

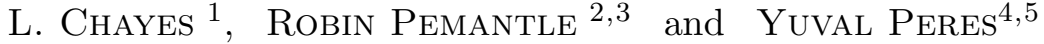

\begin{abstract}
We consider the fractal percolation process on the unit square, with fixed decimation parameter $N$ and level dependent retention parameters $\left\{p_{k}\right\}$; that is, for all $k \geq 1$, at the $k$ th stage every retained square of side-length $N^{1-k}$ is partitioned into $N^{2}$ congruent subsquares, and each of these is retained with probability $p_{k}$, independently of all others. We show that if $\prod_{k} p_{k}=0$ (i.e., if the area of the limiting set vanishes a.s.) then a.s. the limiting set contains no directed crossings of the unit square (a directed crossing is a path that crosses the unit square from left to right, and moves only up, down and to the right).
\end{abstract}

Keywords : Fractal percolation, Oriented percolation, Branching process in varying environment.

Subject classification : Primary: 60K35; secondary: 82B43, 60J80

\footnotetext{
${ }^{1}$ Department of Mathematics, University of California, Los Angeles, California 90024.

${ }^{2}$ Research supported in part by National Science Foundation grant \# DMS 9300191, by a Sloan Foundation Fellowship, and by a Presidential Faculty Fellowship.

${ }^{3}$ Department of Mathematics, University of Wisconsin, Madison, WI 53706 .

${ }^{4}$ Research partially supported by NSF grant \# DMS 9404391

${ }^{5}$ Mathematics Institute, The Hebrew University, Jerusalem, Israel and Department of Statistics, University of California, Berkeley, CA .
} 


\section{Introduction}

Determining the most regular curves that are contained in the planar Brownian path $B[0,1]$ is a well-known open problem (see Duplantier et al (1988)); even the intuitively "obvious" statement that $B[0,1]$ does not contain line segments is not easy to prove (see Pemantle 1995) and it is not known whether $B[0,1]$ contains oriented paths or directed paths.

Since this problem has so far been intractable, in this paper we consider a "mean field approximation" to it, where the Brownian path $B[0,1]$ is replaced by a random fractal with more statistical independence, arising from "fractal percolation".

Fractal percolation is a process that generates a sequence $\left\{A_{k}\right\}$ of random subsets of $[0,1]^{2}$. Let $\left\{p_{k}\right\}_{k \geq 1}$ be a sequence of numbers in $(0,1]$ and let $N \geq 2$ be an integer. The unit square, denoted $A_{0}$, is partitioned into $N^{2}$ congruent squares each of which is independently retained with probability $p_{1}$ or discarded with probability $\left(1-p_{1}\right)$. The closure of the retained, or surviving, squares constitutes the set $A_{1}$. For $k>1$ The set $A_{k} \subset A_{k-1}$ is generated by repeating this procedure, appropriately rescaled, on all the surviving squares of $A_{k-1}$, using the parameter $p_{k}$. The limiting set defined by $A_{\infty}=\bigcap_{k} A_{k}$ is the principal focus of study. The numbers of retained squares of different sizes form a branching process in a varying environment; the offspring distribution at the $k$ th generation is $\operatorname{Binomial}\left(N^{2}, p_{k}\right)$ (see figure 1 ).

In the usual version of the model, the probabilities $p_{k}$ are identically equal to some $p \in(0,1)$. This is too crude for our purpose, since it yields random fractals of Hausdorff dimension less than 2; to obtain a better approximation to the Brownian path (which has dimension 2), we will consider $p_{k} \rightarrow 1$; this yields a limit set $A_{\infty}$ of Hausdorff dimension 2 (a.s. when $A_{\infty}$ is nonempty).

For the case of constant retention probabilities $p_{k}=p$, Chayes, Chayes and Durrett (1988) showed that $\inf _{k} \mathbf{P}\left\{A_{k}\right.$ contains a left-to-right crossing path $\}>0$, provided that $p$ is close enough to 1 .

For the standard percolation model in $\mathbf{Z}^{d}$, when the parameter $p<1$ is large enough there is a.s. an infinite open north-east oriented path (see Durrett 1984). Recently, L. Chayes (1995) 


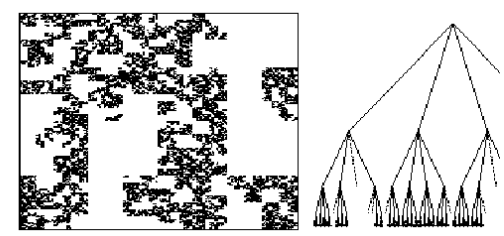

Figure 1: $A_{\infty}$ and the corresponding branching process.

showed that for fractal percolation with fixed $p<1$, there are a.s. no north-east oriented crossings of the unit square in $A_{\infty}$, and furthermore, there are no directed crossings.

Definition. Let $\Gamma:[0,1] \mapsto[0,1]^{2}$ be a continuous path, and write $\Gamma(t)=\left(\gamma_{1}(t), \gamma_{2}(t)\right)$. The image $\Gamma[0,1]$ is called a (left-to-right) directed crossing of the unit square if $\gamma_{1}(0)=0$, the function $\gamma_{1}$ is nondecreasing, and $\gamma_{1}(1)=1$.

Here we establish a stronger result:

Theorem 1.1 Consider the fractal percolation process in $[0,1]^{2}$ with decimation parameter $N \geq 2$ and retention parameters $\left\{p_{k}\right\}$. If $\prod_{k=1}^{\infty} p_{k}=0$, then a.s. $A_{\infty}$ does not contain a directed crossing of the unit square.

The condition $\prod_{k} p_{k}=0$ is clearly equivalent to the area of $A_{\infty}$ vanishing a.s., but this equivalence is not used in the proof of the theorem. The strategy of our proof is to exhibit many nearly horizontal "contours" (defined in the next section) that cannot be crossed by a 
directed path in $A_{\infty}$. These contours force any directed crossing of the unit square in $A_{\infty}$ to be nearly horizontal; a simple counting argument precludes nearly horizontal crossings.

The maximal height of the lowest contour at resolution $N^{-r}$ is bounded by introducing the smallest vacancies first, and estimating the effect of larger vacancies by repeated application of the following lemma:

Lemma 1.2 Let $Y$ be an exponential random variable with parameter $\theta$, i.e., $\mathbf{P}[Y>y]=e^{-\theta y}$ for $y>0$. Then for any random variable $Z \geq 0$ which is independent of $Y$ and has finite mean,

$$
\mathbf{E}[\min \{Z, Y\}] \leq \frac{2 \mathbf{E} Z}{2+\theta \mathbf{E} Z}
$$

Remark. This inequality can also be used to relate electrical conductance to expected maximum flow in a random network; see Proposition 4.1.

\section{Proof of Theorem 1.1}

It will be convenient to extend the fractal percolation process to the half-strip $[0,1] \times[0, \infty)$ by generating, in each of the squares $[0,1] \times[m-1, m]$ for $m \in \mathbb{N}$, an independent copy of the process as described in the introduction.

Definitions. Let $k \geq 0$. A square $S$ of the form $\left[(j-1) N^{-k}, j N^{-k}\right] \times\left[(\ell-1) N^{-k}, \ell N^{-k}\right]$, where $1 \leq j \leq N^{k}$ and $\ell \geq 1$, is called a ( $k$ th level) commensurate square.

In this section, it is convenient to construct $A_{r}$ in a different fashion than in the introduction. Let $\Xi_{k}$ denote a random subset of the collection of level- $k$ commensurate squares, where each level- $k$ square is in $\Xi_{k}$ with probability $1-p_{k}$, independently of all others. Then $A_{r}$ is the union of all level- $r$ commensurate squares that are not contained in any square from $\cup_{k=1}^{r} \Xi_{k}$.

A contour over the interval $[a, b] \subset[0,1]$ is a sequence of squares $S_{1}, \ldots S_{M}$, each of which is in $\cup_{k=1}^{\infty} \Xi_{k}$, such that

(i) the right edge of $S_{i}$ lies on the same line as the left edge of $S_{i+1}$ for $i=1, \ldots, M-1$; 
(ii) the top of $S_{i+1}$ is at or above the same height as the bottom of $S_{i}$ for $i=1, \ldots, M-1$;

(iii) the left edge of $S_{1}$ and the right edge of $S_{M}$ are on the lines $x=a$ and $x=b$ respectively.

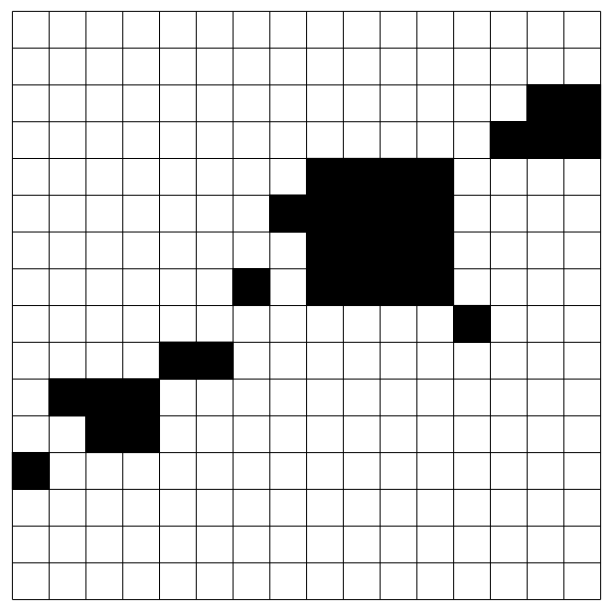

Figure 2: A contour over $[0,1]$.

The contour is called a $(k, r)$-contour if all the squares comprising it are in $\cup_{j=k}^{r} \Xi_{j}$. The bottom edges of the squares in a contour form the graph of a step function; the maximum of this function on the interval $[a, b] \subset[0,1]$ is called the height of the contour over $[a, b]$.

Proposition 2.1 Fix an integer $r \geq 2$. Let $\theta_{k}=\log \left(1 / p_{k}\right)$ and $\Theta_{k}=\sum_{j=1}^{k} \theta_{j}$ for all $k$. For $k \leq r$, denote by $H_{k}$ the minimum, among all $(k, r)$-contours over $\left[0, N^{-k}\right]$, of their height over that interval. Then

$$
\mathbf{E}\left[H_{k}\right] \leq \frac{2 N^{-k}}{\Theta_{r}-\Theta_{k-1}} \text { for all } k \leq r .
$$

Moreover, the minimum, among all $(k, r)$-contours over [0,1], of their height over $[0,1]$, has mean bounded above by $\frac{2}{\Theta_{r}-\Theta_{k-1}}$.

Remark: The contour heights $H_{k}$ that appear in the proposition also depend on $r$, but since $r$ is fixed throughout the proof of the proposition, it is suppressed from the notation. 
To motivate the proof, consider momentarily the cases where $N=2$ and $k$ is either $r$ or $r-1$. Start by examining the process on $\left[0,2^{-r}\right] \times[0, \infty)$ and considering only vacancies of scale $2^{-r}$. The probability of observing a "contour" at height $\ell 2^{-r}$ is evidently $\left(1-p_{r}\right)\left(p_{r}\right)^{\ell}$, so $2^{r} H_{r}$ has expectation $p_{r} /\left(1-p_{r}\right)<\theta_{r}^{-1}$. Now examine the situation on $\left[0,2 \cdot 2^{-r}\right] \times[0, \infty)$, illustrated in Figure 3.

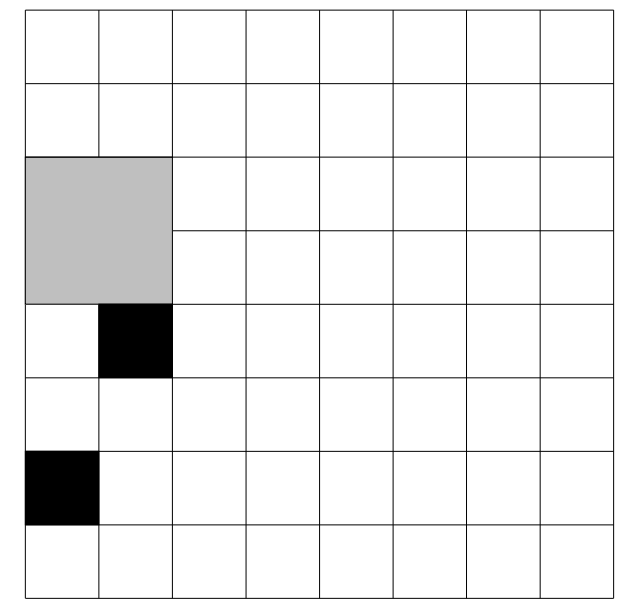

Figure 3: $H_{r-1} \leq \min \left\{\left(H_{r}+H_{r}^{\prime}\right), 2^{1-r} G_{r-1}\right\}$

Taking into account only the vacancies from $\Xi_{r}$, in the first column we find the bottom edge of the lowest vacant square at height $H_{r}$. Assuming that $H_{r}>0$, we may start the second column at height $H_{r}-2^{-r}$, and go up a random height $H_{r}^{\prime}$ until reaching the next vacancy from $\Xi_{r}$, where $H_{r}$ and $H_{r}^{\prime}$ are i.i.d. However, the lowest square in $\Xi_{r-1}$ occurs after $G_{r-1}$ squares of of side $2^{1-r}$, where $\mathbf{P}\left[G_{r-1} \geq \ell\right]=p_{r-1}^{\ell}$ for all $\ell \geq 0$. Recalling that $H_{r-1}$ is the height of the lowest $(r-1, r)$-contour over $\left[0,2^{-r-1}\right]$, we have

$$
H_{r-1} \leq \min \left\{\left(H_{r}+H_{r}^{\prime}\right), 2^{1-r} G_{r-1}\right\}
$$

This inequality can be generalized to arbitrary decimation parameters $N \geq 2$ and to coarser scales, but some care is needed, as for levels $k<r$, contour heights need not be integer multiples of the current scale $N^{-k}$. It is easily checked that $G_{r-1}$ is stochastically dominated by an 
exponential variable with parameter $\theta_{r-1}$. Thus from (2) and Lemma 1.2 we get

$$
\mathbf{E}\left[H_{r-1}\right] \leq \frac{4 \mathbf{E}\left[H_{r}\right]}{2+2^{r-1} \theta_{r-1} \cdot 2 \mathbf{E}\left[H_{r}\right]} \leq \frac{2 \cdot 2^{1-r}}{\theta_{r}+\theta_{r-1}}
$$

a special case of (何).

Proof of Proposition 2.1: Recall that $r \geq 2$ is fixed. For any $v \geq 0$ and $k \leq r$, consider the class of $(k, r)$-contours over $\left[0, N^{-k}\right]$ such that the top of their leftmost square is at height $\geq v$; denote by $H_{k}(v)$ the minimum, among these contours, of their height above the line $y=v$ over the interval $\left[0, N^{-k}\right]$. We will prove that for all $k \leq r$,

$$
\mathbf{E}\left[H_{k}(v)\right] \leq \frac{2 N^{-k}}{\Theta_{r}-\Theta_{k-1}} \text { for all } v \geq 0 .
$$

The proof proceeds inductively starting from $k=r$, and considering the smallest (level- $r$ ) vacancies first. Let $G_{k}(v)$ denote the number of of consecutive level- $k$ squares on the leftmost column before the lowest square in $\Xi_{k}$, counting upward from the level- $k$ square that intersects the line $y=v$. (If there are two such squares, start from the higher one). Let $Y_{k}(v)$ be an exponential variable with parameter $\theta_{k}$. For integer $\ell \geq 0$, clearly

$$
\mathbf{P}\left[G_{k}(v) \geq \ell\right]=p_{k}^{\ell}=e^{-\theta_{k} \ell}=\mathbf{P}\left[\left\lfloor Y_{k}(v)\right\rfloor \geq \ell\right]
$$

so by enlarging the underlying probability space we can couple $G_{k}(v)$ with $Y_{k}(v)$ and assume that $G_{k}(v)=\left\lfloor Y_{k}(v)\right\rfloor$. Thus

$$
H_{k}(v) \leq N^{-k} G_{k}(v) \leq N^{-k} Y_{k}(v) \text { for } 1 \leq k \leq r .
$$

In particular, this gives (3) for $k=r$. For $k<r$ we can concatenate $N(k+1, r)$-contours over intervals of length $N^{-k-1}$ to form a $(k, r)$-contour over $\left[0, N^{-k}\right]$. This leads to the inequality

$$
H_{k}(v) \leq \sum_{i=1}^{N} H_{k+1}^{(i)}\left(v_{i}\right) \text { for } k=0, \ldots, r-1,
$$

where $H_{k+1}^{(i)}(\cdot)$ measures contour heights over the interval $\left[\frac{i-1}{N^{k+1}}, \frac{i}{N^{k+1}}\right]$, and the $v_{i}$ are defined by $v_{1}=v$ and $v_{i+1}=v_{i}+H_{k+1}^{(i)}\left(v_{i}\right)$ (we suppress the dependence of $v_{i}$ on $v, k$ and $r$ from 
the notation). Denote $Z_{k}(v)=\sum_{i=1}^{N} H_{k+1}^{(i)}\left(v_{i}\right)$. Combining (6) and the effect (5) of level- $k$ vacancies, we infer that

$$
H_{k}(v) \leq \min \left\{Z_{k}(v), N^{-k} Y_{k}(v)\right\} \text { for } k=1, \ldots, r-1
$$

Note that $H_{k+1}^{(i)}(\cdot)$ is determined by the collection of squares from $\cup_{\ell=k+1}^{r} \Xi_{\ell}$ in the strip $\left[\frac{i-1}{N^{k+1}}, \frac{i}{N^{k+1}}\right] \times[0, \infty)$, while $v_{i}$ is determined by the collection of squares from $\cup_{\ell=k+1}^{r} \Xi_{\ell}$ in the strip $\left[0, \frac{i-1}{N^{k+1}}\right] \times[0, \infty)$; on the other hand, $G_{k}(v)$ only depends on $\Xi_{k}$, so we may take $Z_{k}(v)$ and $Y_{k}(v)$ to be independent.

The induction hypothesis gives

$$
\mathbf{E} Z_{k}(v)=\sum_{i=1}^{N} \mathbf{E} H_{k+1}^{(i)}\left(v_{i}\right) \leq \frac{2 N^{-k}}{\Theta_{r}-\Theta_{k}} .
$$

By Lemma 1.2 and the inequality (7),

$$
\mathbf{E} H_{k}(v) \leq \frac{2 \mathbf{E}\left[Z_{k}(v)\right]}{2+N^{k} \theta_{k} \mathbf{E}\left[Z_{k}(v)\right]} \leq \frac{2 N^{-k}}{\Theta_{r}-\Theta_{k}+\theta_{k}} .
$$

This suffices to verify (3) by induction.

The last assertion of the proposition follows by concatenating $N^{k}(k, r)$-contours over intervals of length $N^{-k}$.

Proof of Theorem 1.1 completed: We are given that

$$
\Theta_{r}=\sum_{k=1}^{r} \log \left(1 / p_{k}\right) \rightarrow \infty \text { as } r \rightarrow \infty .
$$

In particular

$$
1-p_{k}>N^{-k / 6} \text { for infinitely many } k \text {. }
$$

The probability that $A_{\infty}$ contains a specific point is at most $4 \prod_{k=1}^{\infty} p_{k}=0$, so we may restrict attention to directed crossings that do not contain any point with both coordinates rational. Such a crossing cannot pass through a contour. Fix $k<r$, and consider $(k, r)$-contours over $[0,1]$ that lie above the line $y=\frac{i}{N^{k}}$. Observe that if $S$ is a square commensurate at some level $\geq k$, and its bottom is below the line $y=\frac{i+1}{N^{k}}$, then $S$ must be contained in the rectangle $[0,1] \times$ 
$\left[\frac{i}{N^{k}}, \frac{i+1}{N^{k}}\right]$. Proposition 2.1 implies that the expected height over $[0,1]$ above the line $y=\frac{i}{N^{k}}$ of the lowest such $(k, r)$-contour is at most $\frac{2}{\left(\Theta_{r}-\Theta_{k-1}\right)}$. By Markov's inequality, the probability that the rectangle $[0,1] \times\left[\frac{i}{N^{k}}, \frac{i+1}{N^{k}}\right]$ does not contain a $(k, r)$-contour over $[0,1]$ is at most $\frac{2 N^{k}}{\Theta_{r}-\Theta_{k-1}}$. This is also an upper bound for the probability that the rectangle $[0,1] \times\left[\frac{i-2}{N^{k}}, \frac{i-1}{N^{k}}\right]$ does not contain a reflected ("upside-down") $(k, r)$-contour, provided that $i \geq 2$. If the contour and the reflected contour just mentioned exist, then a vertically aligned triple of squares from $\Xi_{k}$ at any one of $N^{k}$ locations (centered on row $i$ from the bottom) suffices to preclude the existence of a directed crossing that starts in the square $\left[0, \frac{1}{N^{k}}\right] \times\left[\frac{i-1}{N^{k}}, \frac{i}{N^{k}}\right]$ and stays within $A_{r}$ (see Figure 4 ). For $i=1$, existence of the upper contour mentioned above and two vertically aligned squares from $\Xi_{k}$ (in rows 1 and 2) are enough to yield the same conclusion.

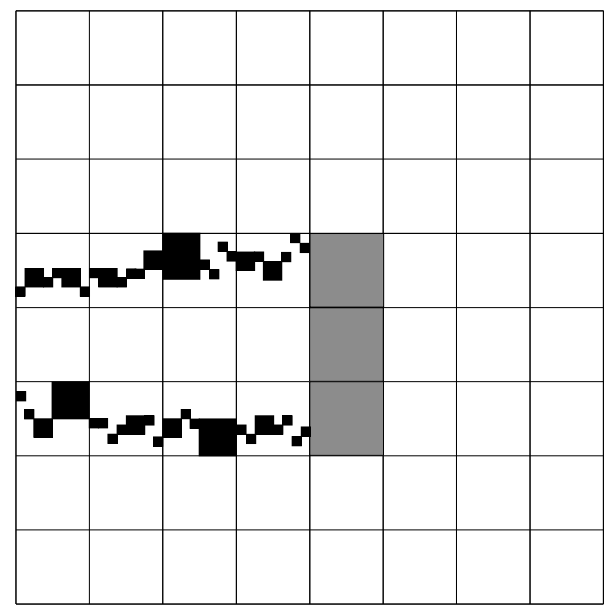

Figure 4: A contour, a reflected contour, and three more vacancies, block directed paths.

Thus the probability that $A_{\infty}$ contains a directed crossing of the unit square is at most

$$
\lim _{r \rightarrow \infty} N^{k}\left(\frac{4 N^{k}}{\Theta_{r}-\Theta_{k-1}}+\left(1-\left(1-p_{k}\right)^{3}\right)^{N^{k}}\right) \leq N^{k} \exp \left(-N^{k}\left(1-p_{k}\right)^{3}\right)
$$

by (8). Finally, by (9), for infinitely many $k$ this probability is bounded by $N^{k} \exp \left(-N^{k / 2}\right)$, so it must vanish. 


\section{Proof of Lemma 1.2}

For $z \geq 0$ we have

$$
\mathbf{E}[\min \{z, Y\}]=\int_{0}^{\infty} \mathbf{P}[\min \{z, Y\}>y] d y=\int_{0}^{z} e^{-\theta y} d y=\frac{1}{\theta}\left(1-e^{-\theta z}\right) .
$$

Hence for any nonnegative random variable $Z$, Jensen's inequality yields

$$
\mathbf{E}[\min \{Z, Y\}]=\frac{1}{\theta} \mathbf{E}\left(1-e^{-\theta Z}\right) \leq \frac{1}{\theta}\left(1-e^{-\theta \mathbf{E} Z}\right) .
$$

Now rewrite the inequality

$$
2+x \geq \sum_{k=0}^{\infty} \frac{2-k}{k !} x^{k}=(2-x) e^{x} \quad \forall x \geq 0
$$

in the equivalent form

$$
1-e^{-x} \leq \frac{2 x}{2+x}
$$

Combining this with (10) proves the lemma.

\section{Concluding remarks}

1. An examination of the proof of Theorem 1.1 has led to the following proposition concerning flow in a random network.

Proposition 4.1 (Lyons, Pemantle and Peres, 1996) Consider a tree $\Gamma$ whose edges are assigned independent exponential random variables $\{Y(e)\}$ with means $\{m(e)\}$. Regard $\{Y(e)\}$ as an assignment of capacities to the edges of $\Gamma$, and let $F$ denote the strength of the maximum flow from the root to the boundary of $\Gamma$ that is bounded by these capacities. On the other hand, let $\mathcal{C}$ be the effective conductance from the root to the boundary for the edge conductances $m(e)$. Then $\mathcal{C} \leq \mathbf{E}[F] \leq 2 \mathcal{C}$.

The maximum flow $F$ can also be represented as a first-passage percolation time in a certain planar dual of the tree; the lower bound $\mathcal{C} \leq \mathbf{E}[F]$ (but not the upper bound) extends to networks that are not trees. 
2. We do not know if the "zero area" condition in Theorem 1.1 is necessary to prevent $A_{\infty}$ from containing directed crossings. In other words, if $\prod_{k=1}^{\infty} p_{k}>0$, does $A_{\infty}$ contain a directed crossing with positive probability?

3. The Brownian path is "intersection-equivalent" to the limiting set $A_{\infty}$ arising from fractal percolation with retention probabilities $p_{k}=k /(k+1)$, see Peres (1996), but unfortunately intersection-equivalence is too weak an equivalence relation to derive any rigorous conclusion about Brownian motion from Theorem 1.1.

\section{References}

[1] L. Chayes (1995). On The Absence of Directed Fractal Percolation, J. Phys. A: Math. Gen. 28, L295-L301.

[2] J. T. Chayes, L. Chayes and R. Durrett (1988). Connectivity Properties of Mandelbrot's Percolation Process, Probab. Th. Rel. Fields 77, 307-324.

[3] B. Duplantier, G. F. Lawler, J.- F. Le Gall and T. J. Lyons (1993). The Geometry of The Brownian curve, Bull. Sci. Math. $2^{e}$ série 117, 91-106.

[4] R. Durrett (1984). Oriented percolation in two dimensions, Ann. Probab. 12, 999-1040.

[5] R. Lyons, R. Pemantle and Y. Peres (1996). Resistance bounds for first-passage percolation and maximum flow. In preparation.

[6] R. Pemantle (1995). The probability that Brownian motion almost contains a line, Ann. Inst. Henri Poincaré, Probab. et Statist., to appear.

[7] Y. Peres (1996). Intersection-equivalence of Brownian paths and certain branching processes. Comm. Math. Phys. 177, 417-434. 